\title{
Assessment of LES for Investigating Spray Flame Impinging on a Wall under Compression-Ignition Engine-like Environment
}

\author{
Abhishek L. Pillai*1, Reo Kai ${ }^{1}$, Jing Li ${ }^{1}$, Ryoichi Kurose ${ }^{1}$ \\ ${ }^{1}$ Department of Mechanical Engineering and Science, Kyoto University, Kyoto, Japan \\ ${ }^{*}$ Corresponding author email: pillai.abhisheklakshman.2e@kyoto-u.ac.jp
}

\begin{abstract}
The applicability of Large-Eddy Simulation (LES) for analysing the phenomenon of turbulent spray flame impinging on a wall, under Compression-Ignition $(\mathrm{Cl})$ engine-like environment is assessed. The fuel spray is $n$-dodecane whose turbulent combustion is modelled using a Non-Adiabatic Flamelet/Progress-Variable (NA-FPV) approach. LESs are based on a EulerianLagrangian framework and the sigma sub-grid turbulence model is used to calculate the subgrid scale (SGS) turbulent viscosity. Dynamics of liquid fuel film formed on the wall surface are captured using a particle-based framework. To couple the convective and radiative heat transfer at the wall surface, with the conduction heat transfer within the finite thickness solid wall, Conjugate Heat Transfer (CHT) is incorporated. LESs are performed with and without SGS models using different mesh resolutions, and the results are compared with those of a Direct Numerical Simulation (DNS). Application of sub-grid turbulence models is found to be crucial for accurate predictions of ignition delay time, flame lift-off length, wall heat flux, and other statistical quantities. It is demonstrated that LES can be successfully applied for reproducing the characteristics of turbulent spray flame, and its interaction with a wall upon impingement, while achieving 5 - 6 times speed-up in computational performance compared to DNS.
\end{abstract}

\section{Keywords}

Large-Eddy Simulation, Wall impinging spray flame, Conjugate Heat Transfer.

\section{Introduction}

Climate change is causing alterations in weather patterns across the globe and triggering catastrophic events, such as hurricanes, typhoons, floods, etc. more frequently. To mitigate the challenges of climate change, many countries have set ambitious new commitments to drastically reduce their $\mathrm{CO}_{2}$ emissions [1]. One of the major contributors to $\mathrm{CO}_{2}$ emissions is the Compression Ignition $(\mathrm{Cl})$ engine (a type of internal combustion engine) widely-used in automobiles. The road transportation sector accounts for roughly $16 \%$ of the global $\mathrm{CO}_{2}$ emissions [2]. Hence, the development of $\mathrm{Cl}$ engines capable of lower emission levels and higher thermal efficiencies is urgently needed. However, this requires the elucidation of heat loss mechanism through the engine cylinder/combustion chamber wall during spray flame-wall interaction. For this purpose, even state-of-the-art experiments cannot provide complete information about the in-cylinder processes, due to physical restrictions of the experimental setup and limitations in the measurement methods. However, Large-Eddy Simulation (LES) seems to be a promising numerical technique that provides quantitative information of the in-cylinder processes, and could be used for analysing such phenomenon, given that it's relatively cheaper and faster to perform compared to experiments. Some recent studies have applied LES for investigating the transient behaviour of spray flames under $\mathrm{Cl}$ engine-like conditions, for example, the ones by Tillou et al. [3] and Pei et al. [4]. But, a thorough assessment of LES for investigating wall impinging spray flame under $\mathrm{Cl}$ engine-like environment, has not yet been performed. Therefore, in this study, 3-D LESs of turbulent spray flame impinging on a wall under $\mathrm{Cl}$ engine-like conditions are performed, with and without sub-grid scale turbulence models using different mesh resolutions, and the results are validated against those of a Direct Numerical Simulation (DNS) 


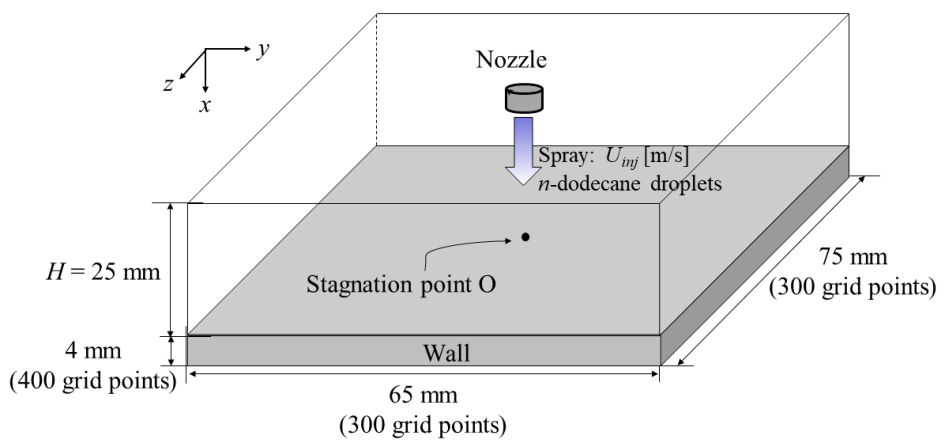

Figure 1. Schematic of the computational domain used in the simulations.

[5], to demonstrate the applicability of LES (and its sub-grid scale models) for predicting ignition delay time, flame lift-off length, wall temperature and heat flux, and other flow-field quantities.

\section{Computational details and model formulation}

The schematic in Figure 1 represents the computational domain used in the LESs. Domain for the computations of spray flame impinging on a wall is discretized by a non-uniform staggered Cartesian grid, with minimum grid spacings of $\Delta x=2.9 \mu \mathrm{m}, \Delta y=\Delta z=60 \mu \mathrm{m}$. For the discretization in the $x$-direction, the grid spacing decreases from the nozzle exit plane to the wall surface. The simulations are based on a previous experiment [5] in which, firstly, premixed $\mathrm{H}_{2}$ air gas was charged into the combustion chamber and ignited. Subsequent flame propagation throughout the chamber created a high pressure and temperature gas mixture at 3.0 MPa and $1600 \mathrm{~K}$, respectively, and comprising $\mathrm{N}_{2}, \mathrm{O}_{2}$ and $\mathrm{H}_{2} \mathrm{O}$, with their respective volume fractions of $0.6,0.21$ and 0.19 . Hence, the same initial ambient gas pressure, temperature and composition are used in the LESs and DNS. Wall thickness is $4 \mathrm{~mm}$ and it is uniformly discretized in the wallnormal $x$-direction using 400 grid points. Initial temperature distribution inside the wall varies linearly from $481 \mathrm{~K}$ on the top surface to $463 \mathrm{~K}$ at the bottom surface (which is kept isothermal), and the wall surface emissivity $\varepsilon$ is assumed to be 1 . Fuel for the liquid spray is $n$-dodecane, and the size distribution of fuel droplets that are injected into the domain from the spray nozzle located at the top, is prescribed using a Rosin-Rammler Probability Density Function (PDF) [5]. Fuel injection parameters used in the LES cases as well as the DNS are the same, and these are summarized in Table 1.

The LESs and DNS of two-phase reacting flows are performed using the conventional EulerianLagrangian framework $[6,7]$, wherein the continuous gas-phase is modelled in the classical Eulerian coordinate framework, while the dispersed-phase fuel droplets are tracked individually in the Lagrangian framework as point-masses. In the LESs, the Favre-filtered conservation equations of mass, momentum, enthalpy, mixture fraction and progress variable are solved for the gas-phase. Evolution of the fuel spray is governed by a set of Lagrangian equations for droplet trajectory, velocity, temperature and mass, which describe the dynamics of individ-

Table 1. Fuel injection parameters (same for all LES cases and DNS).

\begin{tabular}{c|c}
\hline Parameters & Values \\
\hline Fuel injection velocity $[\mathrm{m} / \mathrm{s}]$ & 216 \\
Nozzle diameter $[\mathrm{mm}]$ & 0.083 \\
Fuel injection rate $[\mathrm{mg} / \mathrm{ms}]$ & 0.85 \\
Spray cone angle & 5.16 \\
Sauter Mean Diameter (SMD) $[\mu \mathrm{m}]$ & 10.3 \\
Initial temperature of fuel spray $[\mathrm{K}]$ & 330 \\
\hline
\end{tabular}


ual fuel droplets. Evaporation of fuel droplets is captured via. a non-equilibrium evaporation model based on a Langmuir-Knudsen law [6]. Interactions between gas- and dispersed-phases are coupled using the Particle-Source-In-Cell (PSI-Cell) approach [8]. Details of the governing equations for the gas- and dispersed-phases are explained in Hu and Kurose [9]. The sigma sub-grid turbulence model proposed by Nicoud et al. [10] is used to calculate the sub-grid scale (SGS) turbulent viscosity under the eddy-viscosity assumption. This SGS model is chosen because its formulation promotes the near-wall scaling and vanishing due to turbulence damping properties of the no-slip wall boundary condition. A Non-Adiabatic Flamelet/Progress-Variable (NA-FPV) approach $[11,12]$ wherein the influence of heat loss due to cooled wall, latent heat of evaporation of fuel droplets and liquid fuel film formed on the wall surface, and radiative heat transfer, on the variation of each physical quantity can be accounted for, is employed as the combustion model for $n$-dodecane in the LESs and DNS. The diffusion flamelet database is generated using FlameMaster [13] by solving the flamelet equations for 1-D counter diffusion flame, using a detailed chemical kinetics mechanism of $n$-dodecane consisting of 255 chemical species and 2289 reactions [14]. Spray-film interaction phenomena is captured using the model proposed by Stanton and Rutland [15], and dynamics of the liquid fuel film formed on the wall surface are described using a particle-based numerical approach proposed by O'Rourke and Amsden [16, 17]. Radiative heat transfer is considered using the Discrete Ordinates (DO) method with S8 quadrature set [18], and the dominant radiating entities are soot and gas-phase species, viz. $\mathrm{CO}_{2}, \mathrm{H}_{2} \mathrm{O}$ and $\mathrm{CO}$, all of which are assumed to be gray [19] to keep computational costs realizable. The Hybrid Method of Moments (HMOM) model is employed for computing soot number density function [20] to predict soot formation due to nucleation, coagulation, condensation, surface growth and oxidation. Additionally, Conjugate Heat Transfer (CHT) is incorporated in the LESs and DNS, to couple the convective and radiative heat transfer occurring on the wall surface, with the conduction heat transfer occurring within the solid wall (temperature variation inside the wall is computed by solving the 3-D transient heat conduction equation).

In this study, 4 LES cases using different mesh resolutions far from the wall (maximum grid sizes in the $x$-direction are $90 \mu \mathrm{m}$ and $180 \mu \mathrm{m}$ ), with and without sub-grid turbulence models, are computed to investigate the capability of LES to reproduce turbulent spray flame impinging on a wall. For the cases without sub-grid turbulence models, the SGS terms in the governing equations and the sub-grid variance of mixture fraction are assumed to be zero. Computational details and costs of the LES cases along with those of the DNS are summarized in Table 2. All the simulations are performed using an in-house thermal flow analysis code $\mathrm{FK}^{3}$ [21], which

Table 2. Computational details and predictions of ignition delay time for the LES cases and DNS.

\begin{tabular}{|l|c|c|c|c|c|}
\hline $\begin{array}{l}\text { Simulated Cases } \\
\text { (Cases 1-4 are LESs) }\end{array}$ & Case1 & Case2 & Case3 & Case4 & DNS \\
\hline $\begin{array}{l}\text { Max. grid spacing in } \\
\begin{array}{l}x \text {-direction, } \Delta x_{\max } \\
{[\mu \mathrm{m}]}\end{array}\end{array}$ & 90 & 90 & 180 & 180 & 40 \\
\hline $\begin{array}{l}\text { Sub-grid turbulence } \\
\text { models }\end{array}$ & With & Without & With & Without & - \\
\hline $\begin{array}{l}\text { No. of cores } \\
\text { Wall clock time [hours] }\end{array}$ & 540 & 540 & 540 & 540 & 1600 \\
\hline $\begin{array}{l}\text { CPU hours } \\
\text { Predicted ignition delay } \\
\text { time }[\mu \mathrm{s}]\end{array}$ & 2108,000 & 108,000 & 97,200 & 97,200 & 580,800 \\
\hline $\begin{array}{l}\text { Max. temperature at } \\
\text { ignition delay time }[\mathrm{K}]\end{array}$ & 2237.18 & 2117.08 & 2230.23 & 2157.07 & 2245.54 \\
\hline
\end{tabular}



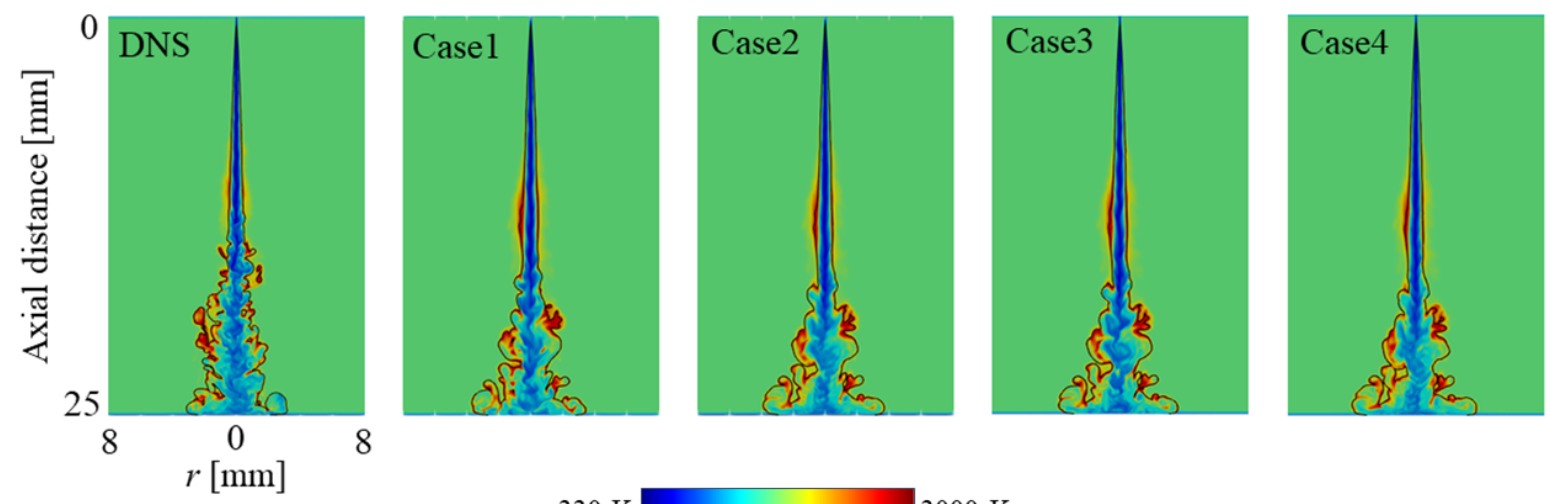

Figure 2. Instantaneous distributions of gas-phase temperature in the central $x-y$ plane for the DNS and LES Cases 1 to 4 , at the time instance of $t=0.294 \mathrm{~ms}$ (time after start of injection).

has been used successfully for simulating a wide variety of gaseous and spray combustion problems, and has also been extensively validated. Additionally, the present DNS has been validated against previous experiment [5]. The CPU time required for each LES case is approximately 0.1 million CPU hours, using MPI parallelization with 540 cores on a CRAY XC40 supercomputer at Kyoto University. Wall-clock time required for the computations with fine and coarse grids are 200 hours and 180 hours, respectively. Hence, the computational costs of the LES cases are $16.74-18.6 \%$ of that of the DNS (roughly $5-6$ times faster than the DNS).

\section{Results and discussion}

Temperature distributions in the central $x-y$ plane obtained from the DNS and 4 LES cases, at the time instance of $0.294 \mathrm{~ms}$ is depicted in Figure 2. At first glance, there seem to be minor differences among the temperature distributions obtained from the DNS and LES cases. However, the trend of temporal evolution of the temperature fields are found to be the same for both the DNS and the LES cases. Significant fluctuations in the temperature field are observed for the DNS prediction compared to the LES cases. This results in different ignition behavior between DNS and LESs. The temporal variations of maximum temperature in the spray flame regions for all cases are shown in Figure 3(a), and it is evident that the temperature rise in DNS is quicker than that in the LES cases. For Cases 2 and 4 (without SGS models), significantly longer ignition times compared to that of the DNS are observed. On the other hand, for Cases 1 and 3 (with SGS models) the ignition delays are quite small in comparison with Cases 2 and 4. Here, ignition delay time is defined as the time from the start of fuel injection to the time when the maximum rate of maximum temperature rise occurs in the domain. The quantitative

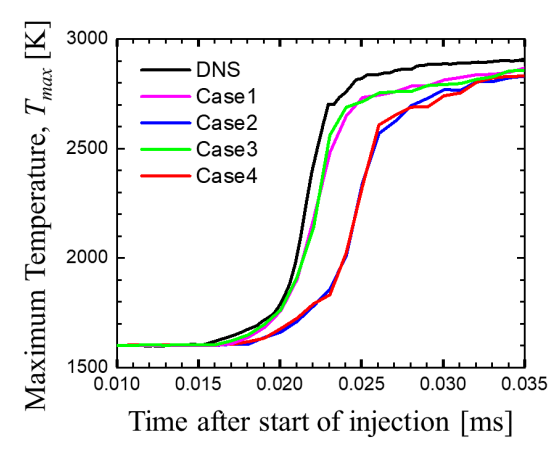

(a)

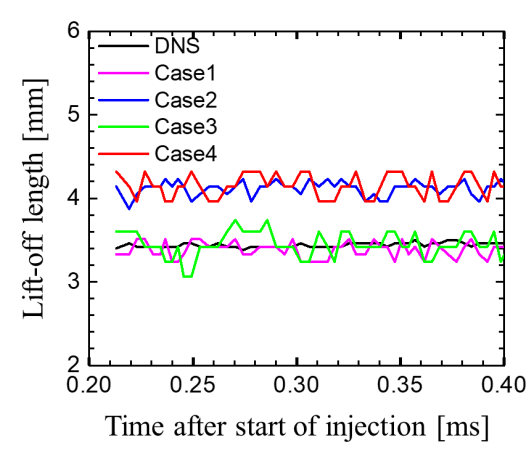

(b)

Figure 3. Comparisons of temporal variations of (a) Maximum gas-phase temperature, $T_{\max }$ in the spray flame region and (b) Lift-off length, among LES Cases 1 to 4 and DNS. 
comparisons of ignition delay time and the maximum temperature at ignition delay time, among the four LES cases and the DNS are also listed in Table 2. There are minor differences in the ignition delay time and the maximum temperature at ignition delay time among Cases 1 and 3, and the DNS. But, these differences are small enough that we consider Cases 1 and 3 to successfully predict the ignition delay time. On the other hand, the ignition delay times predicted by Cases 2 and 4 are longer than that of the DNS. This is mainly because the sub-grid turbulence enhances the mixing process of fuel and oxidizer in Cases 1 and 3. Consequently, sub-grid turbulence increases the mixture fraction in the free shear layer of the spray jet near the nozzle exit, and produces conditions conducive for the flame to ignite earlier in Cases 1 and 3. Furthermore, the small differences in the predicted quantities between Cases 1 and 3 , or Cases 2 and 4 reveal that the influence of mesh resolution on the ignition delay time and the corresponding maximum temperature, is sufficiently small compared to that of sub-grid turbulence models. Figure $3(\mathrm{~b})$ depicts the comparison of the temporal variations of flame liftoff length predicted by the four LES cases and the DNS. Here, flame lift-off length is defined as the axial distance from the nozzle exit to the location of $14 \%$ of the maximum $\mathrm{OH}$ mass fraction corresponding to the quasi-steady state. It is evident that Cases 1 and 3 yield better lift-off length predictions compared to Cases 2 and 4 . The reasons for the large discrepancies in the lift-off lengths predicted by Cases 2 and 4, are the same as those for the ignition delay time predictions stated above.

Following the examinations of ignition delay time and flame lift-off length, some statistics of flow-field quantities are also compared. The axial distributions of time-averaged (quasi-steady state) axial velocity, mixture fraction, temperature and progress variable, in the near-wall region (i.e. for $15 \mathrm{~mm} \leq x \leq 25 \mathrm{~mm}$, where $x=25 \mathrm{~mm}$ is the wall surface) are presented in Figure 4 for all the LES cases along with those obtained from the DNS. Comparing the axial distributions of these quantities among the LES cases and the DNS, it is observed that the predictions of Cases 1 and 3 agree well with that of the DNS. On the other hand, at the axial locations

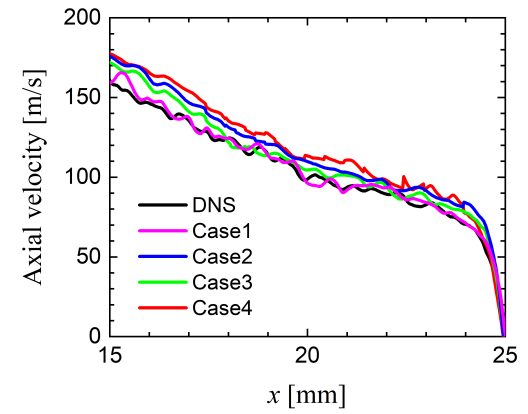

(a)

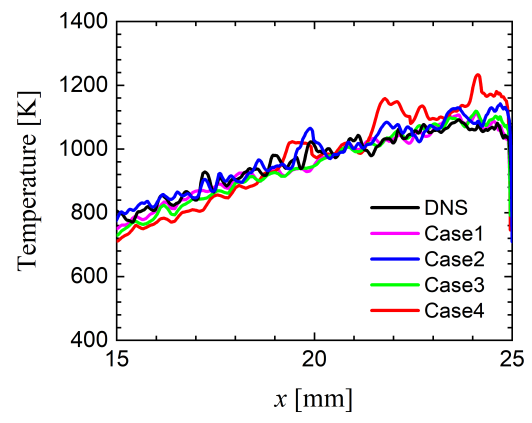

(c)

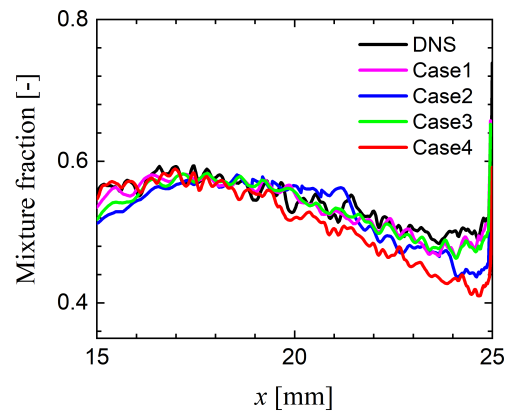

(b)

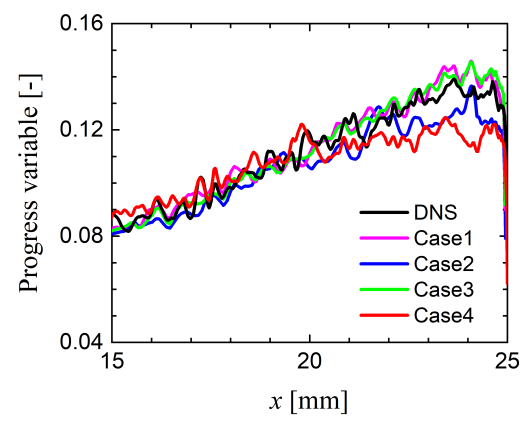

(d)

Figure 4. Comparisons of axial distributions (along the spray nozzle axis) of time-averaged (quasi-steady state) (a) axial velocity, (b) mixture fraction, (c) temperature and (d) progress variable, in the near-wall region among LES Cases 1 to 4 and DNS. 
close to the wall surface, Cases 2 and 4 over-predict the velocity and temperature as shown in Figures 4(a) and 4(c), respectively, and under-predict the mixture fraction and progress variable as shown in Figures 4(b) and 4(d), respectively. The axial distributions of these quantities are not presented for $x<15 \mathrm{~mm}$, because at the upstream axial locations, the results of all the LES cases conform well with those of the DNS. This is because, after the fuel is sprayed out of the nozzle, the gas-phase flow is initially laminar and transitions to turbulent in the downstream direction (towards the wall). Therefore, the influence of sub-grid turbulence is inconsequential in the upstream locations $(x<15 \mathrm{~mm})$. However, the flow becomes increasingly turbulent as the flow approaches closer to the wall, and hence, the sub-grid turbulence models become crucial for accurately predicting the above-mentioned quantities in the near-wall region. Comparing the results of Cases 2 and 4 , it is found that the accuracy of Case $4\left(\Delta x_{\max }=180 \mu \mathrm{m}\right)$ is slightly worse than that of Case $2\left(\Delta x_{\max }=90 \mu \mathrm{m}\right)$. In Figure 5 the time-averaged radial distributions of the same quantities at the streamwise location of $x=21 \mathrm{~mm}$, which is only $4 \mathrm{~mm}$ above the wall surface, are illustrated. As evident from Figures $5(\mathrm{c})$ and $5(\mathrm{~d})$, respectively, the radial distributions of temperature and progress variable predicted by Cases 2 and 4 are different from the DNS results (Case 4's predictions even more so). This observation is in accordance with that for the axial distributions of the flow-field quantities presented in Figure 4. Overall, it can be discerned from Figure 5 that, the radial distributions of all the quantities predicted by Cases 1 and 3 are in much better agreement with the DNS results, and that sub-grid turbulence models are indispensable in the near-wall region (where the flow becomes considerably turbulent) for an accurate reproduction of the spray flame.

Next, the radial distributions of time-averaged (quasi-steady state) conductive heat flux at the wall surface for all the LES cases and DNS are shown in Figure 6(a). It is observed that the radial distribution trend of conductive heat flux, predicted by all the LES cases conform to that

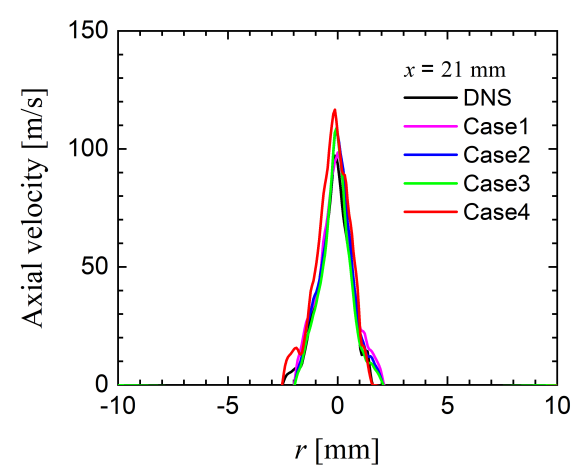

(a)

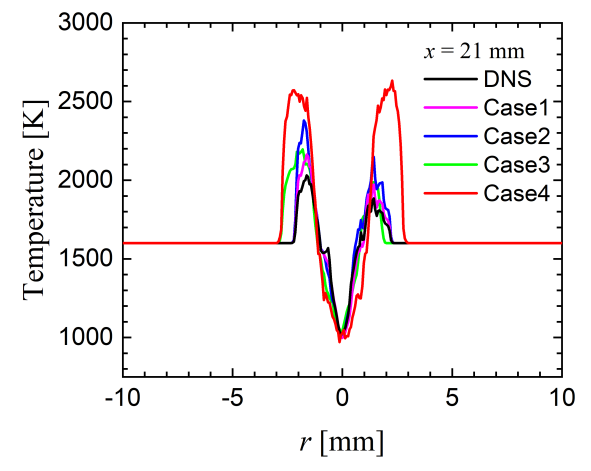

(c)

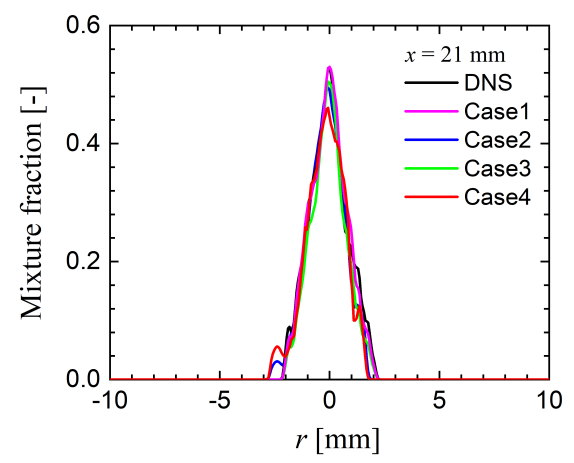

(b)

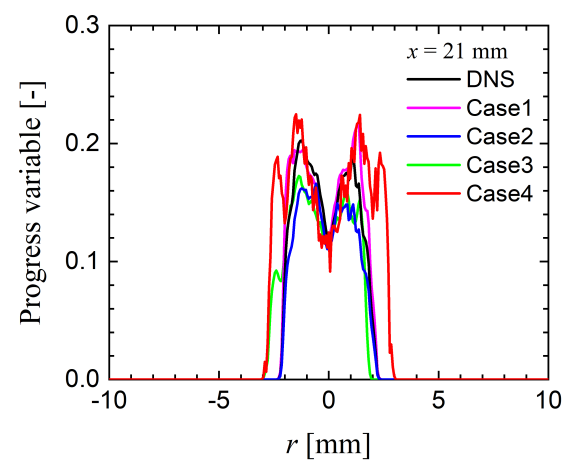

(d)

Figure 5. Comparisons of radial distributions of time-averaged (quasi-steady state) (a) axial velocity, (b) mixture fraction, (c) temperature and (d) progress variable, at the streamwise location of $x=21 \mathrm{~mm}$ (near-wall region) among LES Cases 1 to 4 and DNS. 


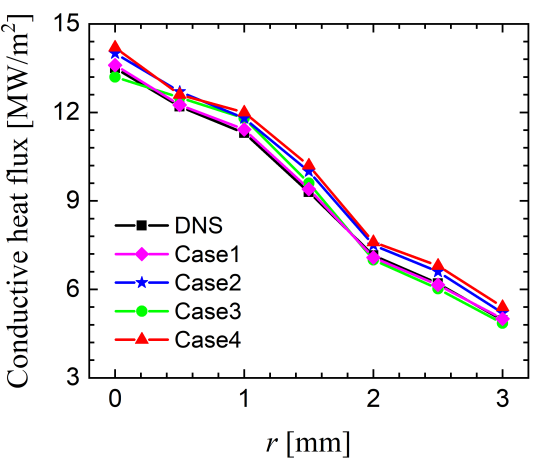

(a)

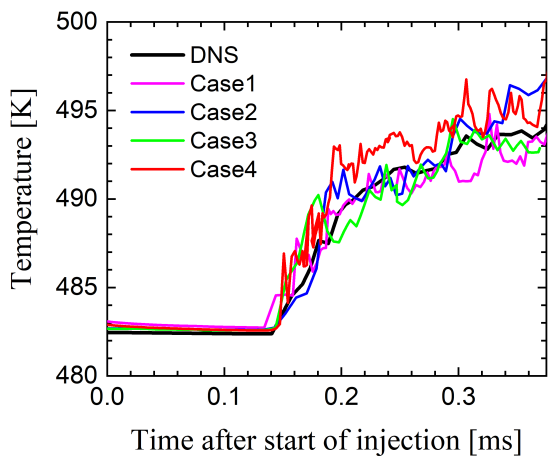

(b)

Figure 6. Comparisons of (a) radial distributions of time-averaged (quasi-steady state) conductive heat flux at the wall surface, and (b) temporal variations of temperature of the stagnation point on the wall surface (i.e. $r=0 \mathrm{~mm}$, point $O$ in Figure 1), among LES Cases 1 to 4 and DNS.

of the DNS, i.e. the conductive heat flux at the wall surface is maximum at the stagnation point $\mathrm{O}(r=0 \mathrm{~mm})$ and decreases as the radial distance from the stagnation point increases. However, by comparing the conductive heat flux at any given radial location in Figure 6(a), it can be seen that Cases 2 and 4 over-predict the conductive wall heat flux compared to DNS. It should be noted that close to the wall surface, the grid spacing in the wall-normal $x$-direction $(\Delta x)$ is the same in all the four LES cases and the DNS. Furthermore, even in Cases 1 and 3 (with sub-grid turbulence models applied), the flow re-laminarizes in the wall jet zone away from the stagnation point, and the sub-grid scale turbulent viscosity will vanish in these laminar flow regions close to the wall surface. Therefore, the discrepancies in the conductive heat flux predicted by Cases 2 and 4 are not caused by the mesh resolution or the lack of subgrid turbulence models in the vicinity of the wall. Rather, these discrepancies arise due to the fact that in Cases 2 and 4, the gas-phase temperatures above the wall surface are higher than those in the DNS and the other two cases (i.e. Cases 1 and 3), as evident from Figures 4(c) and 5 (c). This would subsequently lead to the evolution of higher gas-phase temperatures in the vicinity of the wall surface, and consequently larger temperature gradients at the wall surface in Cases 2 and 4 compared to the other cases. Hence, these larger temperature gradients will in turn induce greater conductive heat flux at the wall surface in Cases 2 and 4 . Therefore, although the mesh resolutions near the wall surface are the same in all the LES cases, the grid size $(\Delta x)$ farther above the wall and the sub-grid turbulence will indirectly influence the convective heat transfer above the wall surface (and hence the conduction heat transfer at the wall surface). Moreover, the time variations of temperature of the stagnation point $O$ on the wall surface shown in Figure 6(b) indicate that, the predictions of Cases 1 and 3 are in good agreement with that of the DNS. Whereas, Cases 2 and 4 (without sub-grid turbulence models) predict higher wall surface temperature at the stagnation point compared to the DNS, which is a direct consequence of the over-predicted conductive heat flux at the wall surface in Cases 2 and 4.

\section{Conclusions}

LESs of a turbulent $n$-dodecane spray flame impinging on a wall under $\mathrm{Cl}$ engine-like conditions were performed by employing a NA-FPV approach, with and without sub-grid turbulence models, and by using different mesh resolutions. Additionally, the dynamics of fuel film formed on the wall surface, radiative heat transfer and conjugate heat transfer were also considered in the LESs. Assessment of LES for analysing such phenomenon was performed by comparing the results of the LES Cases 1-4 with those of DNS. It was found that the mesh resolutions used in the LES cases with sub-grid turbulence models, were adequate to accurately reproduce the 
characteristics of spray flame impinging on a wall. The application of sub-grid turbulence models was also found to be indispensable for accurately predicting the ignition delay time, lift-off length, wall heat flux, temporal variations of wall temperature, and other statistical quantities (such as gas-phase temperature, velocity, mixture fraction and progress variable). Moreover, these quantities were found to be insensitive to the mesh resolution when sub-grid turbulence models are applied. However, the results did not conform to those of DNS when the sub-grid turbulence models were omitted in some LES cases, and the results of those LES cases became worse as the mesh resolution got coarser. Therefore, it was demonstrated in this study that LES can be successfully applied to analyse such phenomenon, while achieving 5 - 6 times faster computational performance than that of the DNS.

\section{Acknowledgements}

This research was partially supported by MEXT as "Program for Promoting Researches on the Supercomputer Fugaku" (Digital Twins of Real World's Clean Energy Systems with Integrated Utilization of Super-simulation and AI), and by JSPS KAKENHI Grant Number $19 \mathrm{H} 02076$.

\section{References}

[1] United Nations Framework Convention on Climate Change, 2015, https : //unfccc.int/process-and-meetings/the-paris-agreement/the-paris-agreement

[2] International Organization of Motor Vehicle Manufacturers, 2019, http://www.oica.net/category/climate-change-and-co2/

[3] Tillou, J., Michel, J. B., Angelberger, C., Veynante, D., 2014, Combustion and Flame, 161 (2), pp. 525-540.

[4] Pei, Y., Som, S., Pomraning, E., Senecal, P. K., Skeen, S. A., Manin, J., Pickett, L. M., 2015, Combustion and Flame, 162, pp. 4442-4455.

[5] Pillai, A. L., March. 17. 2021, Australasian Fluid Mechanics Seminar Series, https://www . youtube.com/watch?v=tZ0i5uQX81M

[6] Miller, R. S., Harstad, K., Bellan, J., 1998, International Journal of Multiphase Flow, 24, pp. 1025-1055.

[7] Pillai, A. L., and Kurose, R., 2019, Combustion and Flame, 200, pp. 168-191.

[8] Crowe, C. T., Sharma, M. P., Stock, D. E., 1977, Journal of Fluids Engineering, 99, pp. 325-332.

[9] Hu, Y., and Kurose, R., 2018, Combustion and Flame, 188, pp. 227-242.

[10] Nicoud, F., Toda, H. B., Cabrit, O., Bose, S., Lee, J., 2011, Physics of Fluids, 23, 085106 (12 pages).

[11] Moriai, H., Kurose, R., Watanabe, H., Yano, Y., Akamatsu, F., Komori, S., 2013, Journal of Engineering for Gas Turbines and Power, 135 (9), 091503 (10 pages).

[12] Kishimoto, A., Moriai, H., Takenaka, K., Nishiie, T., Adachi, M., Ogawara, A., Kurose, R., 2017, Journal of Heat Transfer, 139 (12), 124501 (4 pages).

[13] Pitsch, H., 1998, "A C++ computer program for OD combustion and 1D laminar flame calculations".

[14] Narayanaswamy, K., Pepiot, P., Pitsch, H., 2014, Combustion and Flame, 161, pp. 866884.

[15] Stanton, D. W. and Rutland, C. J., 1996, SAE Transactions, pp. 808-824.

[16] O'Rourke, P. J. and Amsden, A. A., 1996, SAE Transactions, pp. 2000-2013.

[17] O'Rourke, P. J. and Amsden, A. A., 2000, SAE Transactions, pp. 281-298.

[18] Fiveland, W. A., 1984, Journal of Heat Transfer, 106 (4), pp. 699-706.

[19] Chmielewski, M., and Gieras, M., 2015, Journal of Power Technologies, 95 (2), pp. 97-104.

[20] Mueller, M. E., Blanquart, G., Pitsch, H., 2009, Combustion and Flame, 156, pp. 11431155.

[21] Kurose, R., In-house code FK' ${ }^{3}$, http://www.tse.me.kyoto-u.ac.jp/members/kurose/link.php 